\title{
Optical 64QAM-OFDM Transmission Systems with Different Sub-Carriers
}

\author{
Changxiang Li, Yufeng Shao*, Zhifeng Wang*, Junyi Zhou, Yue Zhou, Wenzhe Ma \\ Faculty of Engineering, Shanghai Polytechnic University, Shanghai, China \\ Email: "yfshao@sspu.edu.cn, "zfwang@sspu.edu.cn
}

Received 1 July 2016; accepted 18 August 2016; published 25 August 2016

\begin{abstract}
In this paper, the transceiver performance of optical 64QAM-OFDM signals with different sub-carriers is studied. Firstly, we build a 40Gbit/s optical coherent 64QAM-OFDM transmission system. 64QAM-OFDM optical signals with 16, 32, 64, 64, 128, 256 and 512 sub-carriers are transmitted over $100 \mathrm{~km}$ single mode fiber (SMF). Then, the optical spectrum diagrams before and after transmission, the bit error rate (BER) and constellation diagrams of received signals were compared. The simulation results show that, with the number of sub-carriers increasing, the value of PAPR will gradually increase and the quality of the received optical signals will deteriorate. Moreover, with the number of sub-carriers increasing, the computational complexity will increase when digital signal processing (DSP) is used. Therefore, we should choose the optimal number of sub-carriers, and the PAPR influence and BER are also considered for achieving effective transmission.
\end{abstract}

\section{Keywords}

Optical Fiber Communications, OFDM, Computational Complexity, PAPR, BER

\section{Introduction}

Orthogonal Frequency division multiplex (OFDM) technology has been widely used in the optical transmission systems, because it has characteristics of effectively against dispersion, eliminating the inter symbol interference (ISI) and flexible bandwidth allocation [1] [2]. In OFDM modulating technique, a large number of sub-carriers are multiplexing in the frequency domain, which will cause higher peak to average power ratio (PAPR) [3]. The PAPR not only leads to performance deterioration of linear power amplifier, but also introduces the obvious nonlinear effect [4] [5]. The higher PAPR is one mainly drawback for OFDM signals transmission [6]. In this paper, we analyzed the transceiver performance in coherent 64QAM-OFDM optical transmission system as different number sub-carriers are adopted. Especially, the influence of different number of sub-carriers on the PAPR should be considered. In this paper, we set up one 64QAM-OFDM optical transmission system by simulation. The optical spectral diagrams, the bit error rate (BER) and the constellation diagrams of the received optical coherent 64QAM-OFDM signals in the case of 16, 32, 64, 128, 256 and 512 sub-carriers are measured by simulation, as well as the PAPR and computational complexity are also analyzed.

*Corresponding authors.

How to cite this paper: Li, C.X., Shao, Y.F., Wang, Z.F., Zhou, J.Y., Zhou, Y. and Ma, W.Z. (2016) Optical 64QAM-OFDM Transmission Systems with Different Sub-Carriers. Optics and Photonics Journal, 6, 196-200.

http://dx.doi.org/10.4236/opj.2016.68B032 


\section{System Setup}

Orthogonal Frequency Division Multiplexing technology, the evolution and development of multicarrier modulation (MCM) technology, has been widely attracted much attention [7]. The main idea of OFDM is that divided a high-speed serial data stream into multiple independent low-speed parallel data streams, and then respectively modulated onto different carriers to form more of narrow band data streams transmitted in parallel, to improve the capabilities of data stream against multipath time delay spread [8]-[10].

Figure 1 presents the transmitter end and receiver models for optical coherent 64QAM-OFDM transmission system which this paper mainly studied. Firstly, transmitter data stream should pass the serial to parallel (S/P) converter which converts the serial data stream into a parallel data stream, followed by it is modulated. Then, each sub-carrier is transformed into the time domain waveform by an inverse fast Fourier transform (IFFT). The parallel data stream is then moved back to a serial data stream. The end portion of integrating range is copied into front of the symbol as guard interval to eliminate inter-symbol interference (ISI). After digital-to-analogue conversion, the OFDM modulated signal is transmitted in the form of analog signal.

Figure 2 shown that, we build 64QAM-OFDM simulation system by optisystem software. This paper uses RF to optical (RTO) upconverter to complete the conversion of electrical signal to optical signal. The essence of frequency upconversion is a transformation from complex-value to real-value. RF to optical (RTO) upconverter contains two Mach-Zehnder Modulator to complete real/imaginary parts up-conversion for complex OFDM signal and conversion from optical signal to electrical signal. At the receiver, the system uses two pairs of parallel photo detectors which have $90^{\circ}$ phase displacement. Optical signal is converted to electrical signal by homodyne assays.

\section{Analysis}

In the case of sub-carrier numbers are 128 and 256, we analyzed optical spectral which before and after 64QAM-OFDM signals transmitted over $100 \mathrm{~km}$ single mode fiber and after signals through Gauss filter.

We compared constellations and BER in the case of sub-carriers are 16, 32, 64, 128, 256 and 512. The comparison is shown that the BER is gradually increased and the quality of optical receiving signal gradually deteriorated with the increase of the number of sub-carriers in optical coherent 64QAM-OFDM transmission system.

We compared constellations and BER in the case of sub-carrier numbers are 16, 32, 64, 128, 256 and 512. The comparison shown that the bit error rate is gradually increased and the quality of optical receiving signal gradually deteriorated with the increase of the number of sub-carriers in optical coherent 64QAM-OFDM transmission system.

In the study of PAPR for OFDM signal, we should consider two factors: PAPR and computational complexity. We demonstrated the effect of different sub-carriers for PAPR and computational complexity.

Firstly, we analyzed PAPR of 64QAM-OFDM signal in the case of different sub carriers. PARP is described as the peak to average power ratio, which is a ratio function to measure the size of power fluctuation.

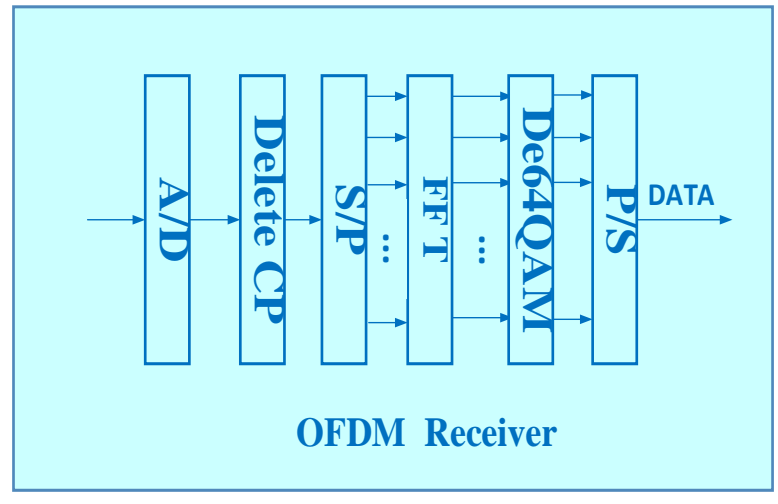

(a)

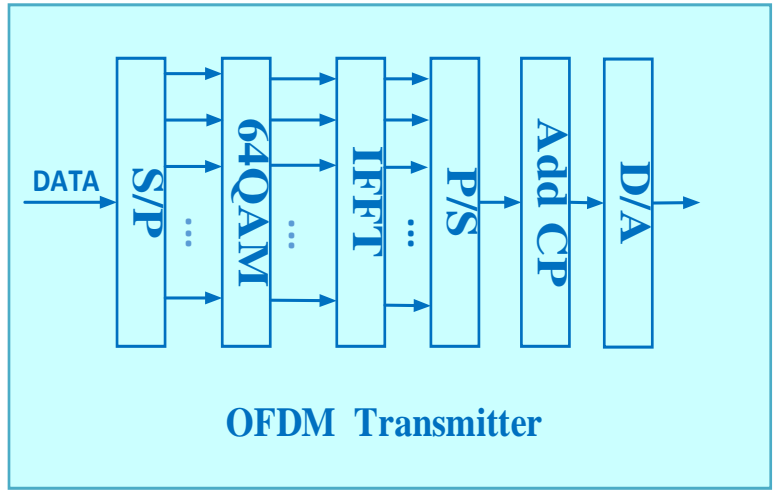

(b)

Figure 1. 64QAM-OFDM (a) transmitter (b) receiver. 


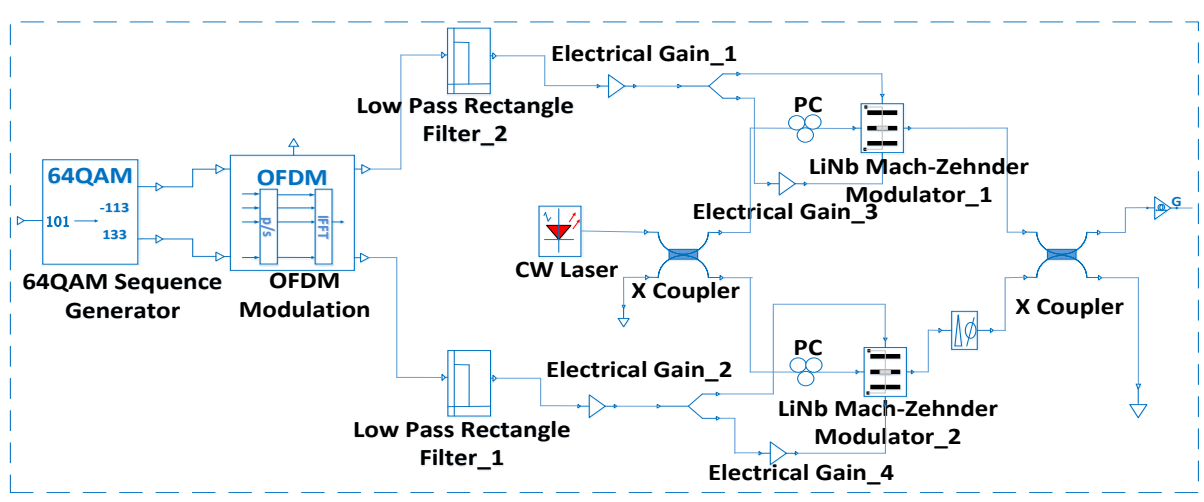

(a)

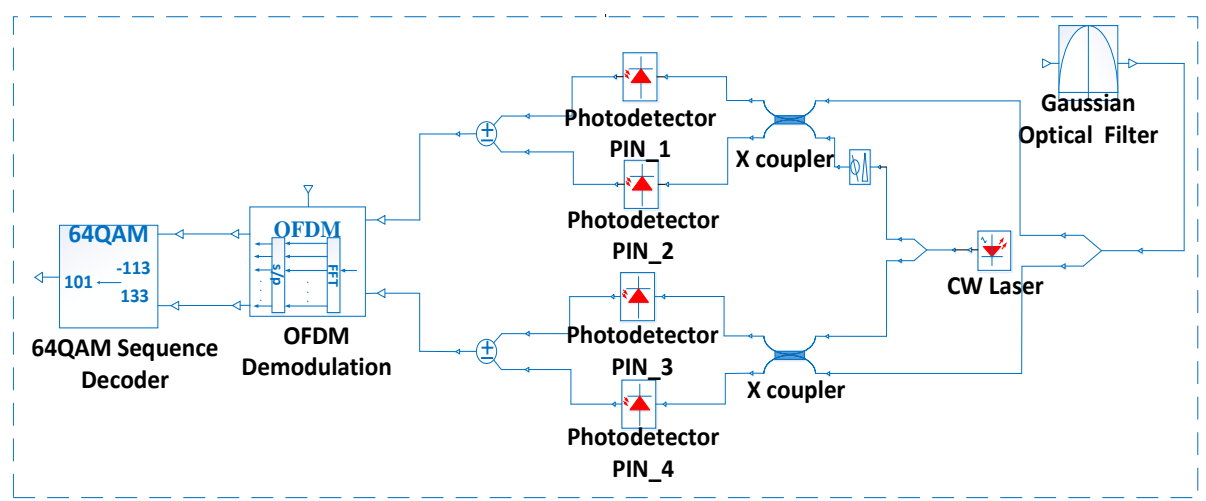

(b)

Figure 2. The structure of optical 64QAM-OFDM (a) transmitter (b) receiver.

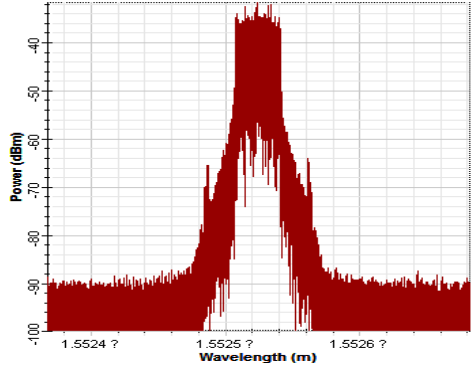

(a)

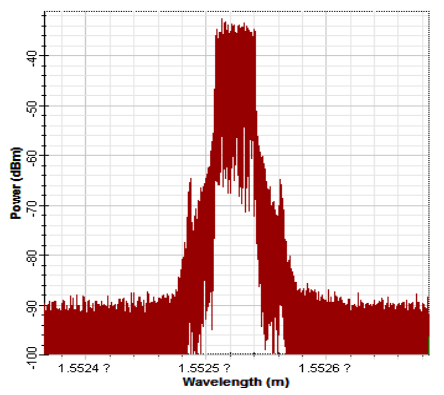

(d)

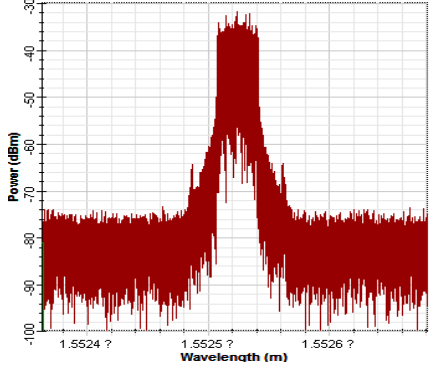

(b)

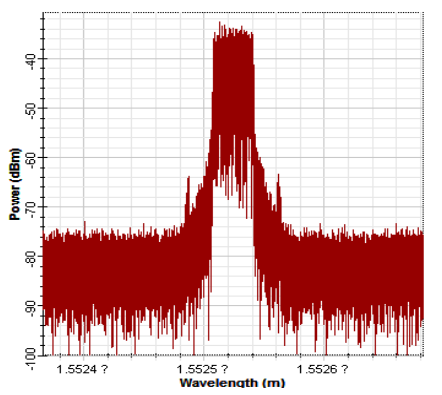

(e)

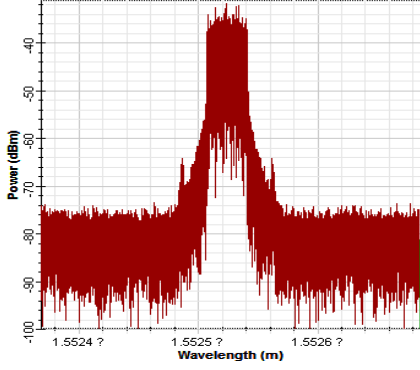

(c)

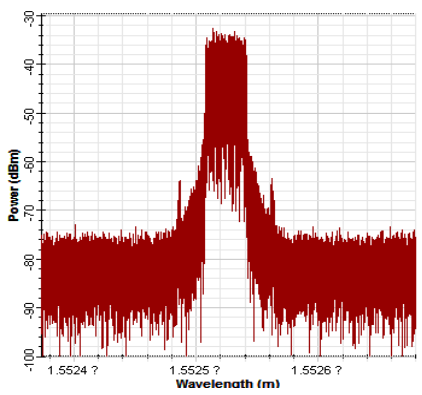

(f)

Figure 3. Optical spectrum diagrams of 128 and 256 sub-carriers. (a) 128 sub-carriers before transmission; (b) 128 sub-carriers after transmission; (c) 128 sub-carriers after filtering; (d) 256 sub-carriers before transmission; (e) 256 sub-carriers after transmission; (f) 256 sub-carriers after filtering. 


$$
\operatorname{PAPR}_{d B}=10 \lg \left\{\frac{\max _{0 \leq t \leq T}|S(t)|^{2}}{\frac{1}{T} \int_{0}^{T}|S(t)|^{2} d t}\right\}, t \in(0, T)
$$

Figure 5(a) described that sub-carriers and PAPR have a positive correlation that peak to average power ratio is increased with the increase of sub-carriers. This requires higher performance to power amplifier and ADC, which results in reduction of efficiency and the cost increase in optical transmission system.

We assume that the sequence length is $N=2^{M}$ and $\mathrm{M}$ is butterfly series of decimation in time (DIT) algorithm. Each butterfly series contain N/2 butterfly operation and a butterfly operation contains a complex multiplication and two complex additions. So, computational complexity of DIT-FFT includes $(N / 2) \log _{2} N$ complex multiplication and $N \log _{2} N$ complex addition. A complex addition can be represented by two real additions and a complex multiplication requires four real multiplications and two real additions to represent. Therefore, a Fourier algorithm requires $2 \operatorname{Nog}_{2} N$ real multiplication numbers and $4 N \log _{2} N$ real addition numbers.

\section{Summary}

Different numbers of sub-carriers in optical coherent 64QAM-OFDM transmission system have impact on tran-

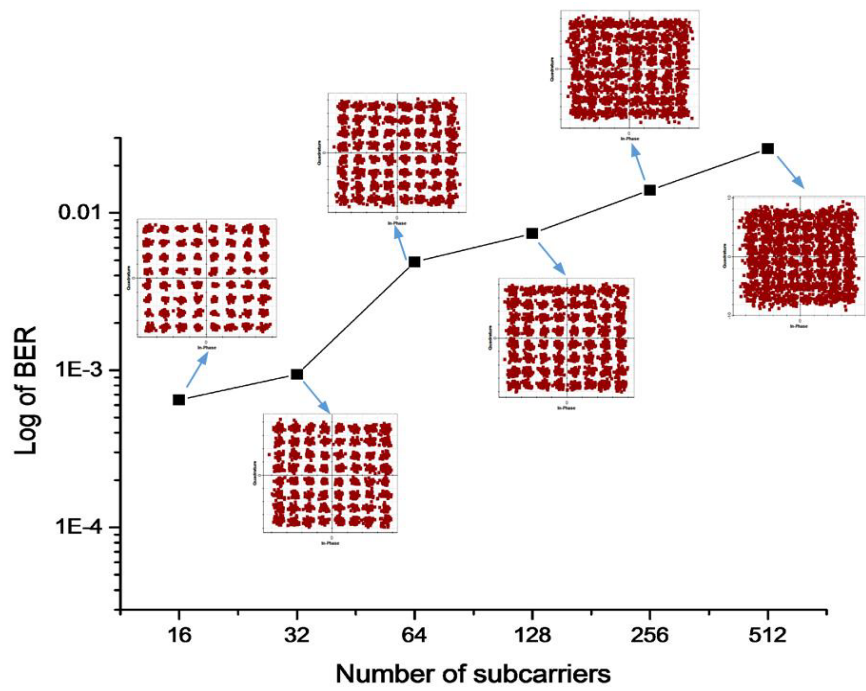

Figure 4. Constellation diagrams and BER of 64QAM-OFDM optical signals with different sub-carriers.

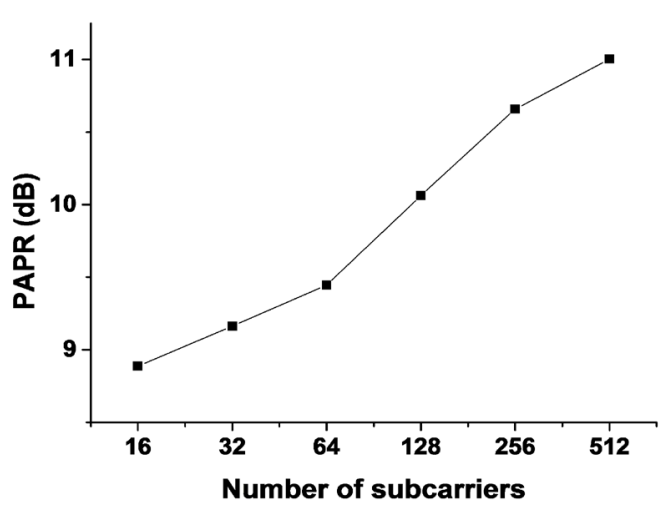

(a)

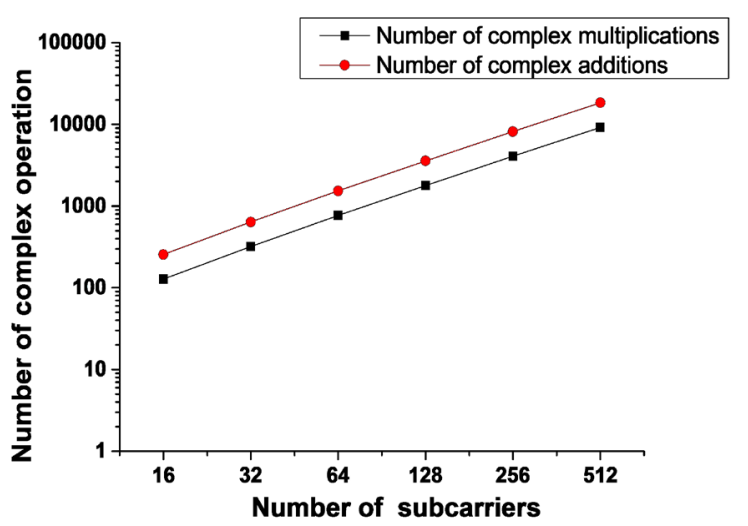

(b)

Figure 5. (a) PAPR and (b) computational complexity of 64QAM-OFDM signals with different sub-carriers. 
sceiver performance. The simulation results show that, the value of PAPR will increase, if the sub-carriers number rise. At the same time, the receiver sensitivity will gradually deteriorate when 64QAM-OFDM optical signals are transmitted over $100 \mathrm{~km} \mathrm{SMF}$. Meanwhile, the number increasing of the sub-carriers will lead to the increase of computational complexity. Therefore, selecting suitable sub-carriers number is important. Moreover, we also should consider the influence of BER, PAPR and computational complexity in high speed OFDM optical transmission systems.

\section{Acknowledgements}

This work is partially supported by the National Natural Science Foundation of China (No. 61107064), Innovation Program of Shanghai Municipal Education Commission (No. 15ZZ101), Leading Academic Discipline Project of Information and Communication Engineering (No.XXKZD1605), School Foundation (No.

EGD14XQD01) of Shanghai Polytechnic University, College Students' Science and Technology Innovation Project of Shanghai Polytechnic University (No. 2016-xjkj-063), and College Student Innovation Activity Plan in Shanghai (No. 2013-sj-cxjh-028).

\section{References}

[1] Shao, Y.F., Wang, Y.J. and Chi, N. (2013) 60-GHZ RoF System with Low PAPR 16QAM-OFDM Downlink Using PTS Segmentation. IEEE Photonics Technology Letters, 25, 855-858. http://dx.doi.org/10.1109/LPT.2013.2252425

[2] Yang, F., He, L.F. and Pan, C.Y. (2013) OFDM Principles and Standards: Evolution of Communication Techniques. Publishing House of Electronics Industry, Beijing, 38-39.

[3] Shao, Y.F., Chi, N. and Fan, J.Y. (2012) Novel Centralized-Light-Source WDM-RoF System with OFDM-CPM Downstream and OOK Upstream. Optics Communications, 285, 3437-3440. http://dx.doi.org/10.1016/j.optcom.2012.03.024

[4] Shao, Y.F., Chi, N., Fan, J.Y. and Fang, W.L. (2012) Generation of 16-QAM-OFDM Signals Using Selected Mapping Method and Its Application in Optical Millimeter-Wave Access System. IEEE Photonics Technology Letters, 24, 1301-1303. http://dx.doi.org/10.1109/LPT.2012.2202387

[5] Shao, Y.F. and Chi, N. (2012) A Novel Scheme for Seamless Integration of RZ-DPSK-DWDM Optical Links with MIMO-OFDM System. Microwave and Optical Technology Letters, 54, 1676-1679. http://dx.doi.org/10.1002/mop.26891

[6] Shao, Y.F., Chen, L., Wen, S.C., Yu, J.J., He, J., Chen, L.L. and Liu, H.Y. (2007) Novel Optical Orthogonally Modulation Scheme for Superimposing DPSK Signals on Dark RZ Signals. Optics Communications, 15, 1415-1416.

[7] Yu, J., Zhou, X., Huang, M.F., Qian, D., Ji, P.N., Wang, T. and Magill, P. (2009) 400Gb/s (4×100Gb/s) Orthogonal PDM-RZ-QPSK DWDM Signal Transmission over 1040km SMF-28. Optics Express, 17, 17928-17933. http://dx.doi.org/10.1364/OE.17.017928

[8] Shao, Y.F., Chi, N., Fan, J.Y. and Fang, W.L. (2012) Generation of 16-QAMOFDM Signals Using Selected Mapping Method and Its Application in Optical Millimeter-Wave Access System. IEEE Photonics Technology Letters, 24, 1301-1303. http://dx.doi.org/10.1109/LPT.2012.2202387

[9] Shao, Y.F., Chi, N., Fan, J.Y., Wang, Y.J. and Fang, W.L. (2012) Novel Centralized-Light-Source WDM-RoF System with OFDM-CPM Downstream and OOK Upstream. Optics Communications, 285, 3437-3440. http://dx.doi.org/10.1016/j.optcom.2012.03.024

[10] Sun, H., Wu, K.-T. and Kim, R. (2008) Real-Time Measurements of a 40 Gb/s Coherent System. Optics Express, 16, 873-879. http://dx.doi.org/10.1364/OE.16.000873 


\section{Submit or recommend next manuscript to SCIRP and we will provide best service for you:}

Accepting pre-submission inquiries through Email, Facebook, LinkedIn, Twitter, etc.

A wide selection of journals (inclusive of 9 subjects, more than 200 journals)

Providing 24-hour high-quality service

User-friendly online submission system

Fair and swift peer-review system

Efficient typesetting and proofreading procedure

Display of the result of downloads and visits, as well as the number of cited articles

Maximum dissemination of your research work

Submit your manuscript at: http://papersubmission.scirp.org/ 